\title{
Amplification of quantum discord between two uncoupled qubits in a common environment by phase decoherence
}

\author{
Ji-Bing Yuan, ${ }^{1}$ Jie-Qiao Liao, ${ }^{1,2}$ and Le-Man Kuang* ${ }^{* \dagger 1}$ \\ ${ }^{1}$ Key Laboratory of Low-Dimensional Quantum Structures and Quantum Control of Ministry of Education, Hunan Normal University, \\ Changsha 410081, China and Department of Physics, Hunan Normal University, Changsha 410081, China \\ ${ }^{2}$ Institute of Theoretical Physics, Chinese Academy of Sciences, Beijing 100190, China
}

(Dated: November 20, 2018)

\begin{abstract}
We study analytically the dynamic behaviors of quantum correlation measured by quantum discord between two uncoupled qubits, which are immersed in a common Ohmic environment. We show that the quantum discord of the two noninteracting qubits can be greatly amplified or protected for certain initially prepared $X$-type states in the time evolution. Especially, it is found that there does exist the stable amplification of the quantum discord for the case of two identical qubits, and the quantum discord can be protected for the case of two different qubits with a large detuning. It is also indicated that in general there does exist a sudden change of the quantum discord in the time evolution at a critic time point $t_{c}$, and the discord amplification and protection may occur only in the time interval $0<t \leq t_{c}$ for certain $X$-type states. This sheds new light on the creation and protection of quantum correlation.
\end{abstract}

PACS numbers: 03.67.-a, 03.65.Ta, 03.65.Yz

\section{INTRODUCTION}

It is well known that the total correlation in a bipartite quantum system can be measured by quantum mutual information [1, 2], which may be divided into classical and quantum parts [2-9]. The quantum part is called quantum discord which is originally introduced by Olliver and Zurek [7]. Recently, it has been aware of the fact that quantum discord is a more general concept to measure quantum correlation than quantum entanglement since there is a nonzero quantum discord in some separable mixed states [7]. In fact, quantum discord is a different type of quantum correlation than entanglement, and it can be considered as a more universal resource than quantum entanglement in some sense. As shown in Refs. [10, 11], although there is no quantum entanglement, quantum discord can also be responsible for the quantum computational efficiency of deterministic quantum computation with one pure qubit [12]. In addition, much recent attention has been paid to many relative topics of quantum discord [1322], such as quantum discord of open quantum systems.

We know that any realistic quantum systems interact inevitably with their surrounding environments, which introduce quantum noise into the systems. As a result, the quantum systems will lose their energy (dissipation) and/or coherence (dephasing). Thus it is of fundamental importance to know the influence of the environment on quantum correlation. In several recent papers [23-27], the quantum correlation dynamics in open quantum systems have been studied. It was shown that the quantum correlation measured by quantum discord is more resistant against the environment than quantum entanglement [23]. For a certain class of states under Markovian dynamics, the quantum entanglement can disappear within a finite time, a phenomenon called by entangle-

\footnotetext{
${ }^{*}$ Author to whom any correspondence should be addressed.

${ }^{\dagger}$ Email: 1mkuang@hunnu.edu.cn
}

ment sudden death [28], which has been widely investigated in recent years [28-33]. Differently, quantum discord only vanishes asymptotically at infinite time [23, 34]. Moreover, for some special initial states, quantum correlation in a bipartite quantum system will not be affected by the decoherence environment during an initial time interval [27].

Based on the above mentioned two facts that quantum discord is a useful resource for quantum information processing, and that quantum systems couple inevitably with their environment. Naturally there are two interesting questions: (i) if the environment can enhance the quantum discord of the systems to realize the discord amplification? (ii) if we can obtain a stable quantum discord induced by the environment? With these questions, in this paper, we study the dynamics of quantum discord between two non-interacting qubits immersed in a common Ohmic environment. We show that it is possible to amplify and protect the quantum discord by the qubit-environment interaction under some conditions. Especially, when the two qubits are identical, we find that the phase decoherence can induce a stable amplification of the initiallyprepared quantum discord for certain $X$-type states.

This paper is organized as follows. In Sec. II we present our physical model and its solution. In Sec.III, we investigate dynamical behaviors of quantum discord for the so-called $X$ type initial states. In Sec. IV we study analytically and numerically the quantum discord for certain $X$-type initial states, and show the initially prepared discord can be amplified or protected for $X$-type states. Finally, we conclude this work in Sec. V.

\section{PHYSICAL MODEL AND SOLUTION}

Let us start with introducing the physical system, as shown in Fig. 1 two uncoupled qubits, qubit $A$ and qubit $B$ with energy separations $\omega_{A}$ and $\omega_{B}$ respectively, are immersed in a common environment. The Hamiltonian of the total system including the two qubits and the environment is composed of 


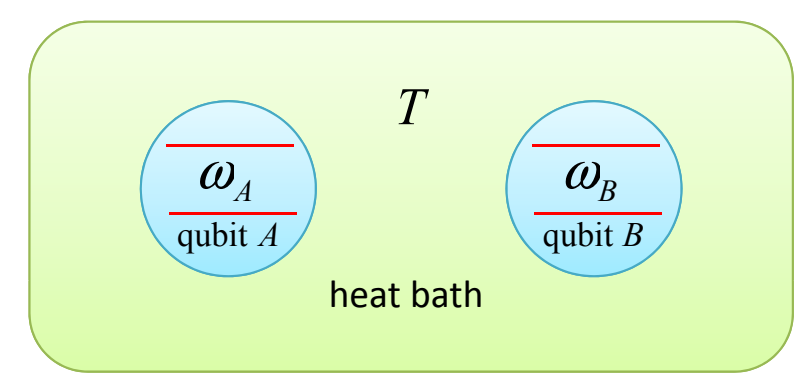

FIG. 1: (Color online) Schematic of our physical system: two uncoupled qubits, of energy separations $\omega_{A}$ and $\omega_{B}$, are immersed in a common heat bath with temperature $T$.

four parts,

$$
\hat{H}_{T}=\hat{H}_{S}+\hat{H}_{E}+\hat{H}_{I}+\hat{H}_{R}
$$

where $\hat{H}_{S}$ is the Hamiltonian of the two qubits, $\hat{H}_{E}$ is the Hamiltonian of the environment, $\hat{H}_{I}$ represents the interaction Hamiltonian between the two qubits and the environment, and $\hat{H}_{R}$ is a renormalization term, which is introduced originally in Ref. [36].

The expression of the Hamiltonian $\hat{H}_{S}$ reads

$$
\hat{H}_{S}=\frac{1}{2} \omega_{A} \hat{\sigma}_{A}^{z}+\frac{1}{2} \omega_{B} \hat{\sigma}_{B}^{z}
$$

where $\hat{\sigma}_{A(B)}^{z}=|0\rangle_{A(B)}\langle 0|-| 1\rangle_{A(B)}\langle 1|$ with $|0\rangle_{A(B)}$ and $|1\rangle_{A(B)}$ being the excited and ground states of the qubit $A(B)$. Hereafter, we take $\hbar=1$.

The environment of the two qubits is modelled by a heat bath with temperature $T$, which is composed of an infinite set of harmonic oscillators with the following Hamiltonian,

$$
\hat{H}_{E}=\sum_{k} \omega_{k} \hat{b}_{k}^{\dagger} \hat{b}_{k}
$$

where $\omega_{k}$ is the frequency of the $k$ th harmonic oscillator depicted by the usual bosonic creation and annihilation operators $\hat{b}_{k}^{\dagger}$ and $\hat{b}_{k}$, satisfying the commutative relation $\left[\hat{b}_{k}, \hat{b}_{k}^{\dagger}\right]=1$.

As for the interaction Hamiltonian $\hat{H}_{I}$ between the two qubits and the heat bath, we assume it is given by the following expression

$$
\hat{H}_{I}=\hat{H}_{S} \sum_{k} g_{k}\left(\hat{b}_{k}^{\dagger}+\hat{b}_{k}\right)
$$

where $g_{k}$ is the coupling constant between the system and the $k$ th harmonic oscillator of the heat bath. It is obvious that $\hat{H}_{I}$ commutes with the Hamiltonian $\hat{H}_{S}$. Therefore there is no energy dissipation in this system. Note that this form of coupling has been used to study quantum decoherence in Bose-Einstein condensation and trapped ion by Kuang and coworkers [37].

The renormalization term has the following form

$$
\hat{H}_{R}=\left(\hat{H}_{S}\right)^{2} \sum_{k} \frac{g_{k}^{2}}{\omega_{k}^{2}} .
$$

According to Ref. [37], Hamiltonian (1) can be exactly solved by making use of the unitary transformation

$$
\hat{U}=\exp \left[\hat{H}_{S} \sum_{k} g_{k}\left(\hat{b}_{k}^{\dagger}-\hat{b}_{k}\right)\right] .
$$

Corresponding to Hamiltonian (1), the total density operator of the system plus the heat bath can be expressed as

$$
\hat{\rho}_{T}(t)=e^{-i \hat{H}_{S} t} \hat{U}^{-1} e^{-i t \sum_{k} \omega_{k} \hat{b}_{k}^{\dagger} \hat{b}_{k}} \hat{U} \hat{\rho}_{T}(0) \hat{U}^{-1} e^{i t \sum_{k} \omega_{k} \hat{b}_{k} \hat{b}_{k}} \hat{U} e^{i \hat{H}_{s} t},
$$

where $\hat{\rho}_{T}(0)$ is the initial total density operator.

We assume the system and the heat bath are initially uncorrelated with the density operator $\hat{\rho}_{T}(0)=\hat{\rho}(0) \otimes \hat{\rho}_{R}(0)$, where $\hat{\rho}(0)$ is the initial density operator of the system, and $\hat{\rho}_{R}(0)$ is the density operator of the heat bath, which is assumed to be $\hat{\rho}_{R}=\prod_{k} \otimes \hat{\rho}_{k}(0)$, where $\hat{\rho}_{k}(0)$ is the density operator of the $k$ th harmonic oscillator in thermal equilibrium of temperature $T$. We can obtain the reduced density operator of the system, denoted by $\hat{\rho}(t)=\operatorname{Tr}_{R}\left[\hat{\rho}_{T}(t)\right]$. Its matrix elements in the eigen representation of $\hat{H}_{s}$ (with the four eigenstates $|00\rangle,|01\rangle,|10\rangle,|11\rangle)$ can be written as

$$
\begin{aligned}
\rho_{\left(l_{A}^{\prime}, l_{B}^{\prime}\right)}\left(l_{A}, l_{B}\right) & (t)= \\
& \rho_{\left(l_{A}^{\prime}, l_{B}^{\prime}\right)\left(l_{A}, l_{B}\right)}(0) R_{\left(l_{A}^{\prime}, l_{B}^{\prime}\right)\left(l_{A}, l_{B}\right)}(t) \\
& \times e^{-i\left[E\left(l_{A}^{\prime}, l_{B}^{\prime}\right)-E\left(l_{A}, l_{B}\right)\right] t},
\end{aligned}
$$

where $E\left(l_{A}, l_{B}\right)\left(E\left(l_{A}^{\prime}, l_{B}^{\prime}\right)\right)$ is the eigenvalue of the operator $\hat{H}_{S}$ with the corresponding eigenstate $\left|l_{A}, l_{B}\right\rangle\left(\left|l_{A}^{\prime}, l_{B}^{\prime}\right\rangle\right)$, the expression of $E\left(l_{A}, l_{B}\right)$ is $E\left(l_{A}, l_{B}\right)=\left[(-1)^{l_{A}} \omega_{A}+(-1)^{l_{B}} \omega_{B}\right] / 2$. The quantity $R_{\left(l_{A}^{\prime}, l_{B}^{\prime}\right)\left(l_{A}, l_{B}\right)}(t)$ is a reservoir-dependent part given by

$$
\begin{aligned}
R_{\left(l_{A}^{\prime}, l_{B}^{\prime}\right)\left(l_{A}, l_{B}\right)}(t)= & e^{-i\left[E^{2}\left(l_{A}^{\prime}, l_{B}^{\prime}\right)-E^{2}\left(l_{A}, l_{B}\right)\right] Q_{1}(t)} \\
& \times e^{-\left[E\left(l_{A}^{\prime}, l_{B}^{\prime}\right)-E\left(l_{A}, l_{B}\right)\right]^{2} Q_{2}(t)} .
\end{aligned}
$$

The two reservoir-dependent functions $Q_{1}(t)$ and $Q_{2}(t)$ in the above Eq. (9) are given by

$$
\begin{aligned}
& Q_{1}(t)=\int_{0}^{\infty} d \omega J(\omega) \frac{g^{2}(\omega)}{\omega^{2}} \sin (\omega t), \\
& Q_{2}(t)=2 \int_{0}^{\infty} d \omega J(\omega) \frac{g^{2}(\omega)}{\omega^{2}} \sin ^{2}\left(\frac{\omega t}{2}\right) \operatorname{coth}\left(\frac{\beta \omega}{2}\right) .
\end{aligned}
$$

Here we have taken the continuum limit of the reservoir modes $\sum_{k} \rightarrow \int_{0}^{\infty} d \omega J(\omega)$, where $J(\omega)$ is the spectral density of the reservoir, $g(\omega)$ is the corresponding continuum expression for $g_{k}$, and $\beta=1 / T$ (with the Boltzmann constant $\left.k_{B}=1\right)$.

\section{DYNAMICS OF QUANTUM DISCORD FOR TWO-QUBIT $X$-TYPE STATES}

In this section, we investigate dynamics of quantum discord for the two non-interacting qubits in the phase decoherence environment for the three-parameter two-qubit $X$-type states. Quantum discord [7] is defined as the difference between the 
total correlation and the classical correlation with the following expression

$$
\mathcal{D}(\hat{\rho})=\mathcal{I}\left(\hat{\rho}_{A}: \hat{\rho}_{B}\right)-C(\hat{\rho}) .
$$

Here the total correlation in a bipartite quantum state $\hat{\rho}$ is measured by quantum mutual information given by

$$
\mathcal{I}\left(\hat{\rho}_{A}: \hat{\rho}_{B}\right)=S\left(\hat{\rho}_{A}\right)+S\left(\hat{\rho}_{B}\right)-S(\hat{\rho}),
$$

where $S(\hat{\rho})=-\operatorname{Tr}(\hat{\rho} \log \hat{\rho})$ is the von Neumann entropy, $\hat{\rho}_{A}=\operatorname{Tr}_{B}(\hat{\rho})$ and $\hat{\rho}_{B}=\operatorname{Tr}_{A}(\hat{\rho})$ are the reduced density operators for subsystems $A$ and $B$, respectively. And the classical correlation between the two subsystems $A$ and $B$ can be defined as

$$
\begin{aligned}
C(\hat{\rho}) & =\max _{\left\{\hat{P}_{k}\right\}}\left[S\left(\hat{\rho}_{A}\right)-\sum_{k} p_{k} S\left(\hat{\rho}_{A}^{(k)}\right)\right] \\
& =S\left(\hat{\rho}_{A}\right)-\min _{\left\{\hat{P}_{k}\right\}}\left[\sum_{k} p_{k} S\left(\hat{\rho}_{A}^{(k)}\right)\right] .
\end{aligned}
$$

Here $\left\{\hat{P}_{k}\right\}$ is a set of projects performed locally on the subsystem $B$, and $\hat{\rho}_{A}^{(k)}=\frac{1}{p_{k}} \operatorname{Tr}_{B}\left[\left(\hat{I}_{A} \otimes \hat{P}_{k}\right) \hat{\rho}\left(\hat{I}_{A} \otimes \hat{P}_{k}\right)\right]$ is the state of the subsystem $A$ conditioned on the measurement of the outcome labelled by $k$, where $p_{k}=\operatorname{Tr}_{A B}\left[\left(\hat{I}_{A} \otimes \hat{P}_{k}\right) \hat{\rho}\left(\hat{I}_{A} \otimes \hat{P}_{k}\right)\right]$ denotes the probability relating to the outcome $k$, and $\hat{I}_{A}$ denotes the identity operator for the subsystem $A$.

In terms of the relation given in Eq. (8), we can study the quantum discord dynamic properties of the two qubits. We assume the two qubits are initially prepared in a class of state with maximally mixed marginals $\left(\hat{\rho}_{A(B)}=\hat{I}_{A(B)} / 2\right)$ described by the three-parameter $X$-type density matrix

$$
\begin{aligned}
\hat{\rho}(0) & =\frac{1}{4}\left(\hat{I}_{A B}+\sum_{i=1}^{3} c_{i} \hat{\sigma}_{A}^{i} \otimes \hat{\sigma}_{B}^{i}\right) \\
& =\frac{1}{4}\left(\begin{array}{cccc}
1+c_{3} & 0 & 0 & c_{1}-c_{2} \\
0 & 1-c_{3} & c_{1}+c_{2} & 0 \\
0 & c_{1}+c_{2} & 1-c_{3} & 0 \\
c_{1}-c_{2} & 0 & 0 & 1+c_{3}
\end{array}\right),
\end{aligned}
$$

where $\hat{I}_{A B}$ is the identity operator in the Hilbert space of the two qubits, $\hat{\sigma}_{A}^{i}$ and $\hat{\sigma}_{B}^{i}(i=1,2,3$ mean $x, y, z$ correspondingly) are the Pauli operators of qubit $A$ and qubit $B$, and $c_{i}$ $\left(0 \leq\left|c_{i}\right| \leq 1\right)$ are real numbers satisfying the unit trace and positivity conditions of the density operator $\hat{\rho}$. The density operator $\hat{\rho}$ includes the Werner states and the Bell states as two special cases. Under the decoherence environment, the evolution of density operator $\hat{\rho}(t)$ initially prepared in Eq. (14) can be obtained according to Eq. (8). Its explicit form at time $t$ is

$$
\hat{\rho}(t)=\frac{1}{4}\left(\begin{array}{cccc}
1+c_{3} & 0 & 0 & \mu e^{i \Delta_{1}} \\
0 & 1-c_{3} & v e^{i \Delta_{2}} & 0 \\
0 & v e^{-i \Delta_{2}} & 1-c_{3} & 0 \\
\mu e^{-i \Delta_{1}} & 0 & 0 & 1+c_{3}
\end{array}\right),
$$

where we have introduced the following parameters

$$
\begin{aligned}
\Delta_{1} & =\left(\omega_{A}+\omega_{B}\right) t, & \Delta_{2} & =\left(\omega_{A}-\omega_{B}\right) t, \\
\mu(t) & =\left(c_{1}-c_{2}\right) \gamma_{1}(t), & v(t) & =\left(c_{1}+c_{2}\right) \gamma_{2}(t), \\
\gamma_{1}(t) & =e^{-\left(\omega_{A}+\omega_{B}\right)^{2} Q_{2}(t)}, & \gamma_{2}(t) & =e^{-\left(\omega_{A}-\omega_{B}\right)^{2} Q_{2}(t) .}
\end{aligned}
$$

As shown in Eq. (16), the decay parameters $\gamma_{1}$ and $\gamma_{2}$ depend on the frequencies $\omega_{A}$ and $\omega_{B}$ of the two qubits and the reservoir-dependent function $Q_{2}$. For convenience, we define a detuning parameter of the two qubits as

$$
r=\frac{\omega_{A}}{\omega_{B}},
$$

which indicates that the detuning parameter $r=1$ for two identical qubits due to $\omega_{A}=\omega_{B}$, and $r \neq 1$ for two different qubits due to $\omega_{A} \neq \omega_{B}$. In other words, the two qubits are resonant (non-resonant) when the detuning parameter $r=1$ $(r \neq 1)$. Then the two decay parameters $\gamma_{1}$ and $\gamma_{2}$ can be connected with each other through the detuning parameter with the following simple expression

$$
\gamma_{2}=\gamma_{1}^{\left(\frac{r-1}{r+1}\right)^{2}} .
$$

When the two qubits are identical, i.e., $r=1$, from Eq. (16) we have $\Delta_{2}=0, \gamma_{2}=1$, and $v=\left(c_{1}+c_{2}\right)$. In this case, equation (15) indicates that there does exist a decoherencefree subspace with two basis states $|1,0\rangle_{A B}$ and $|0,1\rangle_{A B}$ for the $X$-type initial states under present consideration.

In order to obtain the mutual information of state $\hat{\rho}(t)$ given in Eq. (15), we first calculate the four eigenvalues of $\hat{\rho}(t)$,

$$
\lambda_{1,2}=\frac{1}{4}\left(1+c_{3} \mp \mu\right), \quad \lambda_{3,4}=\frac{1}{4}\left(1-c_{3} \mp v\right) .
$$

Then the mutual information reads

$$
\mathcal{I}\left(\hat{\rho}_{A}: \hat{\rho}_{B}\right)=2+\sum_{i=1}^{4} \lambda_{i} \log \lambda_{i} .
$$

Note that here we have used $S\left(\hat{\rho}_{A}(t)\right)=S\left(\hat{\rho}_{B}(t)\right)=1$, since the two reduced density matrixes $\hat{\rho}_{A}(t)$ and $\hat{\rho}_{B}(t)$ are maximally mixed, that is $\hat{\rho}_{A}(t)=\hat{I}_{A} / 2$ and $\hat{\rho}_{B}(t)=\hat{I}_{B} / 2$.

For calculation of the amount for the classical correlation $C(\hat{\rho})$ defined in Eq. (13), we propose the complete set of orthogonal projectors $\left\{\hat{P}_{k}=\left|\theta_{k}\right\rangle\left\langle\theta_{k}\right|, k=\|, \perp\right\}$ for a local measurement performed on the subsystem $B$, where the two projectors are defined in terms of the following two orthogonal states

$$
\begin{aligned}
\left|\theta_{\|}\right\rangle & =\cos \theta|0\rangle+e^{i \phi} \sin \theta|1\rangle, \\
\left|\theta_{\perp}\right\rangle & =e^{-i \phi} \sin \theta|0\rangle-\cos \theta|1\rangle,
\end{aligned}
$$

with $0 \leq \theta \leq \pi / 2$ and $0 \leq \phi \leq 2 \pi$. After the two project measurements with $p_{\|}=p_{\perp}=1 / 2$, the reduced density matrices of subsystem $A$ read

$$
\begin{aligned}
& \hat{\rho}_{A}^{\prime \prime}=\frac{1}{4}\left(\begin{array}{cc}
2\left(1-c_{3} \cos (2 \theta)\right) & \epsilon \sin (2 \theta) \\
\epsilon^{*} \sin (2 \theta) & 2\left(1+c_{3} \cos (2 \theta)\right)
\end{array}\right), \\
& \hat{\rho}_{A}^{\perp}=\frac{1}{4}\left(\begin{array}{cc}
2\left(1+c_{3} \cos (2 \theta)\right) & -\epsilon \sin (2 \theta) \\
-\epsilon^{*} \sin (2 \theta) & 2\left(1-c_{3} \cos (2 \theta)\right)
\end{array}\right),
\end{aligned}
$$

where we have introduced the parameter $\epsilon=\mu e^{i\left(\Delta_{1}-\phi\right)}+$ $v e^{i\left(\Delta_{2}+\phi\right)}$. According to Eq. (22), it is straightforward to obtain the eigenvalues of the reduced density matrix $\hat{\rho}_{A}^{(k)}$ as follows:

$$
\zeta_{1,2}^{(k)}=\frac{1}{2}(1 \pm \Lambda),
$$


where we have defined $\Lambda$ as

$$
\begin{aligned}
\Lambda= & \left\{c_{3}^{2} \cos ^{2}(2 \theta)+\frac{1}{4}\left[\mu^{2}+v^{2}+2 \mu \nu \cos \left(\Delta_{2}-\Delta_{1}+2 \phi\right)\right]\right. \\
& \left.\times \sin ^{2}(2 \theta)\right\}^{\frac{1}{2}}
\end{aligned}
$$

Since $\zeta_{1,2}^{(\|)}=\zeta_{1,2}^{(\perp)}$, then we have $S\left(\hat{\rho}_{A}^{\|}\right)=S\left(\hat{\rho}_{A}^{\perp}\right)=f(\Lambda)$ defined by

$$
f(\Lambda)=-\frac{1-\Lambda}{2} \log _{2}\left(\frac{1-\Lambda}{2}\right)-\frac{1+\Lambda}{2} \log _{2}\left(\frac{1+\Lambda}{2}\right),
$$

which leads to the classical correlation

$$
C(\hat{\rho}(t))=1-\min _{\theta, \phi}[f(\Lambda)]
$$

Since the function $f(\Lambda)$ is a monotonically decreasing function, therefore for getting the minimal value of $S\left(\hat{\rho}_{A}^{\|}\right)=f(\Lambda)$, we should choose proper parameters $\theta$ and $\phi$ to ensure the parameter $\Lambda$ defined in Eq. (24) is maximal. Obviously, from Eq. (24) we can see that the maximal value depends on $c_{3}, \mu$, and $v$. From Eq. (24) it is easy to get the following inequality

$$
\begin{aligned}
\Lambda & \leq\left[c_{3}^{2} \cos ^{2}(2 \theta)+\frac{(|\mu|+|v|)^{2}}{4} \sin ^{2}(2 \theta)\right]^{1 / 2} \\
& \leq \begin{cases}\left|c_{3}\right|, & \text { for }\left|c_{3}\right|>(|\mu|+|v|) / 2, \\
(|\mu|+|v|) / 2, & \text { for }\left|c_{3}\right|<(|\mu|+|v|) / 2 .\end{cases}
\end{aligned}
$$

If we define $\chi(t)$ as

$$
\chi(t)=\max \left[\left|c_{3}\right|,(|\mu(t)|+|v(t)|) / 2\right]
$$

then the classical correlation can be expressed as

$$
C(\hat{\rho}(t))=\sum_{n=1}^{2} \frac{1+(-1)^{n} \chi}{2} \log _{2}\left[1+(-1)^{n} \chi\right] .
$$

Therefore, the quantum discord can be written as

$$
\mathcal{D}(\hat{\rho}(t))=2+\sum_{i=1}^{4} \lambda_{i} \log _{2} \lambda_{i}-C(\hat{\rho}(t)),
$$

where the amount of the classical correlation $C(\hat{\rho}(t))$ is given by Eq. 29). In principle, we have obtained the dynamics of the quantum discord according the above expression given in Eq. (30), provided that we know the initial condition of the system. In what follows we will study dynamic properties of the quantum discord for some initial states in detail.

\section{QUANTUM DISCORD AMPLIFICATION}

In the section, we would like to find the possibility of quantum discord enhancement induced by phase decoherence in the dynamic evolution of the $X$-type quantum states. From Eq. (16) we can see that both $\mu(t)$ and $v(t)$ are two decaying functions with respect to the evolution time $t$ due to the reservoir function $Q_{2}(t)$ being a increase function indicated below, hence there may exist a critic time $t_{c}$. At this time, the following equation may be satisfied

$$
\frac{\left|\mu\left(t_{c}\right)\right|+\left|v\left(t_{c}\right)\right|}{2}=\left|c_{3}\right|
$$

which is the equation to determine $t_{c}$. Obviously, the critic time $t_{c}$ depends on initial-state and reservoir parameters.

From Eqs. (16) and (28) we can obtain

$$
\chi(t)= \begin{cases}\left|c_{3}\right|, & \text { for } t>t_{c}, \\ (|\mu(t)|+|v(t)|) / 2, & \text { for } t<t_{c},\end{cases}
$$

which indicates that the classical correlation expressed by Eq. (29) exhibits a sudden change at the critic time $t_{c}$ determined by Eq. (31). The classical correlation decays monotonically from the initial time to the critic time $t_{c}$, and then keeps constant after the critic time $t_{c}$. Then making use of Eqs. (29-32) we can obtain expressions of the quantum discord in different regimes of the time evolution

$$
\begin{aligned}
& \mathcal{D}(\hat{\rho}(t))=2+\sum_{i=1}^{4} \lambda_{i} \log _{2} \lambda_{i}-\frac{1}{2} \sum_{n=1}^{2} \Lambda_{n}^{\prime} \log _{2} \Lambda_{n}^{\prime}, \quad t<t_{c} \\
& \mathcal{D}(\hat{\rho}(t))=2+\sum_{i=1}^{4} \lambda_{i} \log _{2} \lambda_{i}-\frac{1}{2} \sum_{n=1}^{2} \Lambda_{n} \log _{2} \Lambda_{n}, \quad t \geq t_{c}
\end{aligned}
$$

where $\Lambda_{n}$ and $\Lambda_{n}^{\prime}$ are given by

$$
\Lambda_{n}=1+(-1)^{n}\left|c_{3}\right|, \quad \Lambda_{n}^{\prime}=1+(-1)^{n} \frac{|\mu|+|v|}{2} .
$$

From Eqs. 33a and 33b we can see that there does exist a critic time at which the quantum discord evolution exhibits a sudden change. Therefore, dynamic evolution of the quantum discord may have different behaviors in the time interval $0 \leq t \leq t_{c}$ and after the critic time. In what follows, we shall investigate dynamic characteristics of the quantum discord in the time evolution for two identical and different qubits, respectively.

\section{A. The case of two identical qubits}

For the sake of simplicity, we first consider the case of two identical qubits and assume that the three initial-state parameters obey $c_{2}=0$ and $c_{1}>c_{3}>0$. In this case, we have $\omega_{A}=\omega_{B}=\Omega, r=1$ and $\gamma_{2}=1$. From Eq. (31) we find that the critic time $t_{c}$ satisfies the equation

$$
Q_{2}\left(t_{c}\right)=\frac{1}{4 \Omega^{2}} \ln \left(\frac{2 c_{3}}{c_{1}}-1\right),
$$

where $Q_{2}(t)$ is the reservoir function defined in Eq. (10) which is determined by the spectral density of the environment $J(\omega)$. We consider the Ohmic reservoir [38] with the spectral density $J(\omega)$ given by

$$
J(\omega)=\frac{\eta \omega}{g^{2}(\omega)} \exp \left(-\frac{\omega}{\omega_{c}}\right)
$$




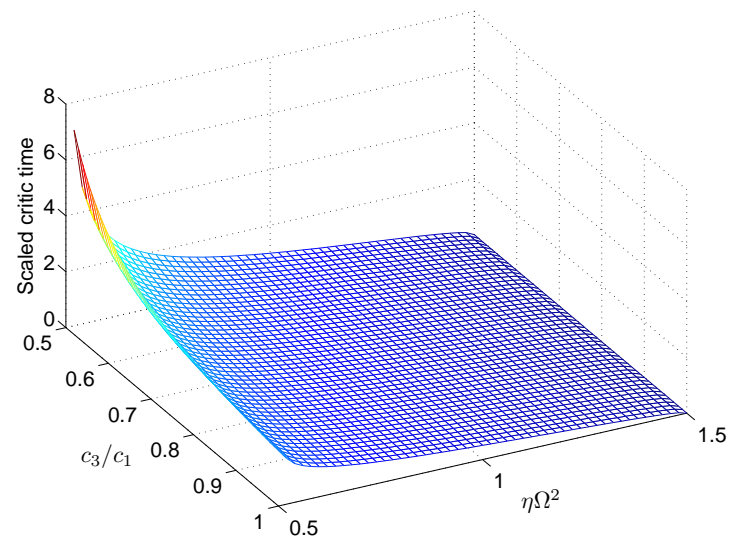

FIG. 2: (Color online) Plot of the scaled critic time $\omega_{c} t_{c}$ given in Eq. (38) vs the qubit parameter $\eta \Omega^{2}$ and the initial-state parameter $c_{3} / c_{1}$ at zero temperature.

where $\omega_{c}$ is the high-frequency cutoff, and $\eta$ is a positive characteristic parameter of the reservoir. With this choice, at low temperature the function $Q_{2}(t)$ is given by the following expression

$$
Q_{2}(t)=\eta\left\{\frac{1}{2} \ln \left[1+\left(\omega_{c} t\right)^{2}\right]+\ln \left[\frac{\beta}{\pi t} \sinh \left(\frac{\pi t}{\beta}\right)\right]\right\} .
$$

The the critic time $t_{c}$ can be obtained from Eqs. (35) and (37). However, it should be pointed out that there does exist the critic time only for certain values of the three initialstate parameters $\left(c_{1}, c_{2}, c_{3}\right)$, not for arbitrary values of these three parameters. Take into account the positivity of the density operator and the reservoir function given by Eq. (37), we find that there exists the critic time $t_{c}$ for some initial states with $0<c_{1} / 2 \leq c_{3}<c_{1} \leq 2 / 3$ and $c_{2}=0$. It is a difficult task to get an analytical expression of the critic time $t_{c}$ for an arbitrary reservoir temperature. However, at zero temperature, from Eqs. (35) and (37) it is straightforward to get the analytic result of the critic time given by

$$
t_{c}=\frac{1}{\omega_{c}} \sqrt{\left(2 c_{3} / c_{1}-1\right)^{-1 /\left(2 \eta \Omega^{2}\right)}-1}
$$

From Eq. (38) we can see that the critic time $t_{c}$ depends on not only the characteristics of the two qubits and their environment but also the initial-state parameters. For a set of given parameters $\left(c_{1}, c_{3}, \eta, \Omega\right)$, the lower is the cut-off frequency of the reservoir, the longer is the critic time. In particular, when $2 c_{3}=c_{1}$ we have $t_{c}=\infty$. This means that the quantum discord will not exhibit the sudden change in the whole process of the time evolution. In Fig. 2 2 we display the critic time with respect to the parameters $\eta \Omega^{2}$ and $c_{3} / c_{1}$ at zero temperature. In the following we show that at zero temperature it is possible to realize the discord amplification in the case of $2 c_{3}=c_{1}$ for the two identical qubits.

For the two identical qubits, when $2 c_{3}=c_{1}$ and $c_{2}=0$ the critic time approaches the infinity. Then the quantum discord

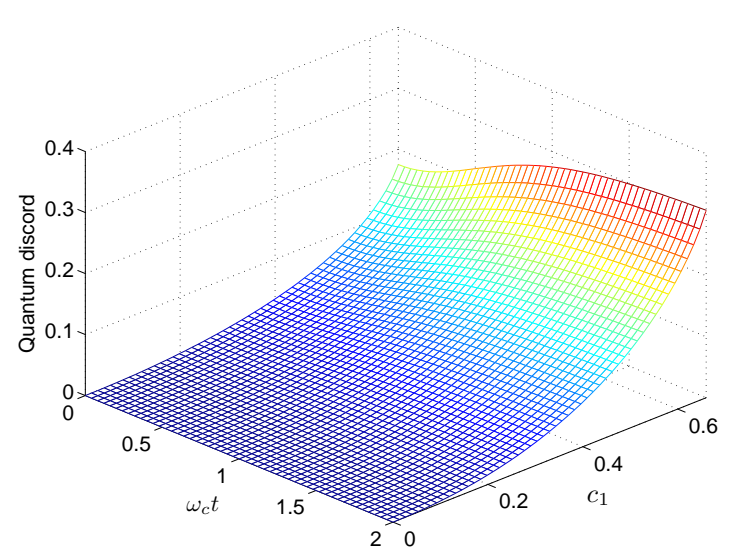

FIG. 3: (Color online) Plot of the quantum discord $\mathcal{D}_{i d}(t)$ for the two identical qubits vs the scaled time $\omega_{c} t$ and the initial-state parameter $c_{1}$ at zero temperature when $\eta \Omega^{2}=1$.

is given by

$$
\mathcal{D}_{i d}(t)=2+\sum_{i=1}^{4} \lambda_{i} \log _{2} \lambda_{i}-\frac{1}{2} \sum_{n=1}^{2} \Lambda_{n}^{\prime} \log _{2} \Lambda_{n}^{\prime}, \quad 0 \leq t<\infty,
$$

where $\lambda_{i}, \Lambda_{n}^{\prime}$, and $\gamma_{1}(t)$ are given by

$$
\begin{aligned}
\lambda_{1,2} & =\frac{1}{8}\left[2+c_{1}\left(1 \mp 2 \gamma_{1}(t)\right)\right], & \lambda_{3,4} & =\frac{1}{8}\left[2-c_{1}(1 \pm 2)\right], \\
\Lambda_{1,2}^{\prime} & =1 \mp \frac{1}{2} c_{1}\left(1+\gamma_{1}(t)\right), & \gamma_{1}(t) & =e^{-4 \Omega^{2} Q_{2}(t)},
\end{aligned}
$$

where the temperature-dependent reservoir function $Q_{2}(t)$ is given by Eq. (37).

In Fig. 3 we have plotted the quantum discord $\mathcal{D}_{i d}(t)$ with respect to the initial-state parameter $c_{1}$ in the time evolution. Figure 3 clearly indicates the amplification of the initial discord in the time evolution. And the discord amplification becomes more apparent for large values of $c_{1}$. In particular, when the time approaches to the infinity, we have $\gamma_{1}=0$, hence the quantum discord reaches its maximal value given by

$$
\begin{aligned}
\mathcal{D}_{i d}(\infty)= & \frac{2+c_{1}}{8} \log _{2}\left(2+c_{1}\right)-\frac{2-c_{1}}{4} \log _{2}\left(2-c_{1}\right) \\
& +\frac{2-3 c_{1}}{8} \log _{2}\left(2-3 c_{1}\right)
\end{aligned}
$$

which implies that the interaction between the reservoir and the two identical qubits can lead to the stable amplification of the initially prepared discord. And the stable value of the quantum discord depends on only the initial-state parameters. In Fig. 4 we display the stable discord $\mathcal{D}_{i d}(\infty)$ with respect to the initial-state parameter $c_{1}$. From Fig. (4) we can see that the larger is the initial-state parameter $c_{1}$, the more apparent the amplification of the quantum discord.

In order to calculate the amplification rate of the quantum discord, we need the quantum discord of the corresponding 


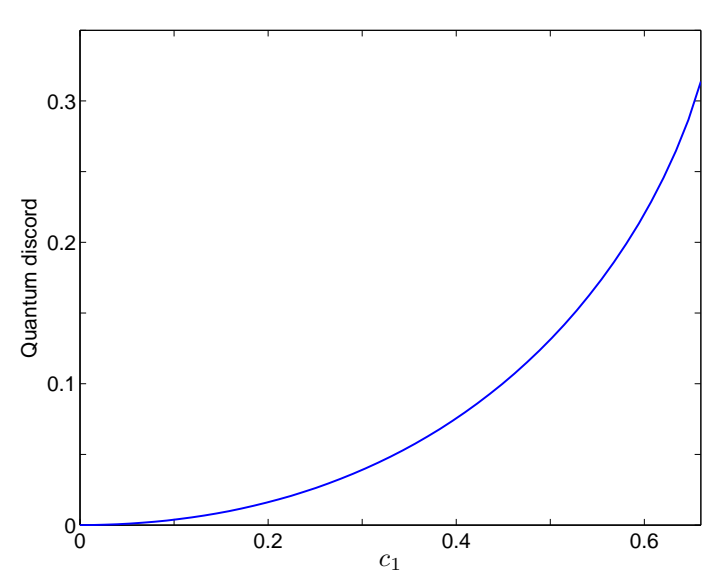

FIG. 4: (Color online) Plot of the quantum discord given in Eq. (41) with respect to the initial-state parameter $c_{1}$.

initial states with the following expression

$$
\begin{aligned}
\mathcal{D}(0)= & -1+\frac{2-c_{1}}{8} \log _{2}\left(2-c_{1}\right)+\frac{2+c_{1}}{8} \log _{2}\left(2+c_{1}\right) \\
& +\frac{2+3 c_{1}}{8} \log _{2}\left(2+3 c_{1}\right)+\frac{2-3 c_{1}}{8} \log _{2}\left(2-3 c_{1}\right) \\
& -\frac{1+c_{1}}{2} \log _{2}\left(1+c_{1}\right)-\frac{1-c_{1}}{2} \log _{2}\left(1-c_{1}\right) .
\end{aligned}
$$

Then we define the amplification rate of the quantum discord as $\Gamma=\mathcal{D}_{i d}(\infty) / \mathcal{D}(0)$. In Fig. 5 we have plotted the amplification rate $\Gamma$ with respect to the initial-state parameter $c_{1}$. Figure 5 indicates that the initially-prepared discord under present consideration can be amplified for the whole regime in which $c_{1}$ can takes its values, and the amplification rate is larger in the middle regime than in other regimes for the values of $c_{1}$. In particular, we can obtain the maximal amplification rate $\Gamma_{\max } \approx 2.17$ when the initial-state parameter $c_{1} \approx 3.3$.

Physically, the stable amplification phenomenon of the quantum discord for the two resonant qubits in the common heat bath is related to the existence of a decoherence-free subspace of the two qubits in the time evolution. In fact, from Eq. (15) we can see that when the two qubits are resonant, i.e., $r=1$, we have $v(t)=0$, the subspace formed by $|0,1\rangle_{A B}$ and $|0,1\rangle_{A B}$ is a decoherence-free subspace of the two qubits in the time evolution. Therefore, the stable amplification phenomenon of the quantum discord can be understood as a quantum coherent effect of the two qubits in the common heat bath.

\section{B. The case of two different qubits}

Dynamic evolution of the quantum discord for two uncoupled different qubits in a common environment can be studied through analyzing the first derivative of the quantum discord with respect to time. In this case, we have $r \neq 1$ due to $\omega_{A} \neq \omega_{B}$. We now discuss the general conditions to realize the amplification of the quantum discord by phase decoherence. Note that the time dependence of the quantum discord

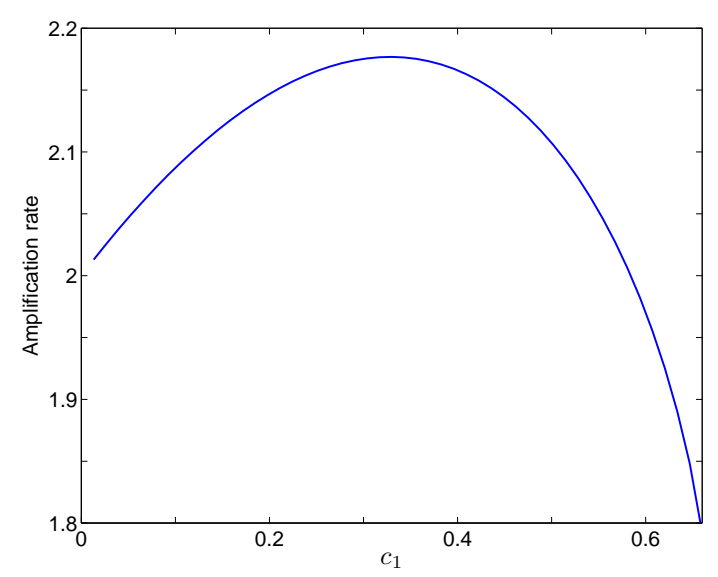

FIG. 5: (Color online) Plot of the amplification rate of the quantum discord $\Gamma=\mathcal{D}_{i d}(\infty) / \mathcal{D}(0)$ with respect to the initial-state parameter $c_{1}$.

given by Eq. (33) can be described in terms of only one parameter $\gamma_{1}(t)$ since the other time-dependent parameters $\lambda_{i}$, $\Lambda_{n}^{\prime}$, and $\gamma_{2}(t)$ can be expressed by $\gamma_{1}(t)$ through the relations given by Eqs. (16) and (17). For the Ohmic-reservoir case under our consideration and at low temperature, making use of Eq. (36) we can find $\gamma_{1}(t)$ to be

$$
\gamma_{1}(t)=\left\{\left[1+\left(\omega_{c} t\right)^{2}\right]^{1 / 2} \frac{\beta}{\pi t} \sinh \left(\frac{\pi t}{\beta}\right)\right\}^{-\eta(r+1)^{2} \omega_{B}^{2}} .
$$

From Eq. (44), it is easy to see that the first derivative of $\gamma_{1}$ with respect to time $t$ is always negative, i.e.,

$$
\frac{\partial \gamma_{1}}{\partial t}<0
$$

which means that the parameter $\gamma_{1}$ monotonically decreases with respect to time $t$. In fact, $\gamma_{1}=\gamma_{2}=1$ at the beginning and $\gamma_{1} \rightarrow 0, \gamma_{2} \rightarrow 0$ at infinite time.

Hence, the derivative of the quantum discord with respect to evolution time can be expressed as

$$
\frac{\partial \mathcal{D}(\hat{\rho}(t))}{\partial t}=\frac{\partial \mathcal{D}(\hat{\rho}(t))}{\partial \gamma_{1}} \frac{\partial \gamma_{1}}{\partial t},
$$

which implies that when

$$
\frac{\partial \mathcal{D}(\hat{\rho}(t))}{\partial \gamma_{1}}<0
$$

and making use of Eq. (45) we can find

$$
\frac{\partial \mathcal{D}(\hat{\rho}(t))}{\partial t}>0
$$

which indicates that the quantum discord is enhanced with the time evolution. This is the condition of the amplification of the quantum discord. In the following we will further study the amplification condition. 


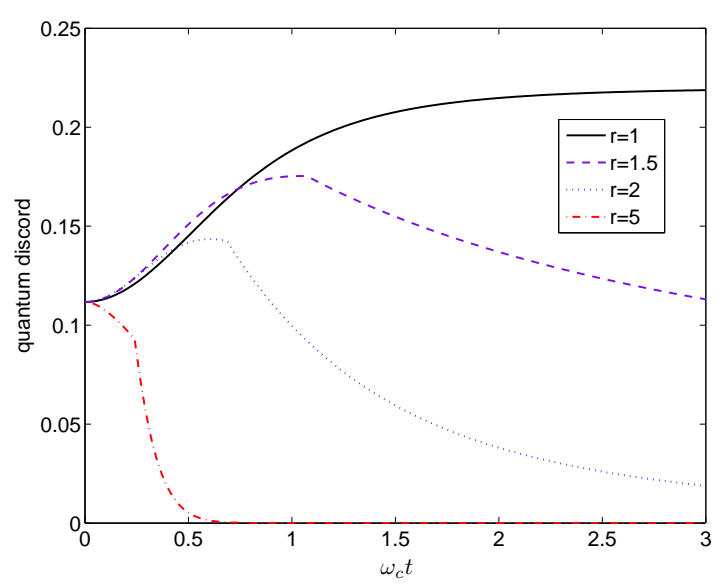

FIG. 6: (Color online) Plot of the quantum discord given in Eq. 33. vs the scaled time $\omega_{c} t$ at zero temperature for different values of the detuning parameter $r: r=1$ (solid black line), $r=1.5$ (dashed purple line), $r=2$ (dotted blue line), and $r=5$ (dot-dashed red line). Other parameters are set as $c_{1}=0.6, c_{2}=0$, and $c_{3}=0.3$.

In order to demonstrate the possibility of the discord amplification, we calculate the first derivative of quantum discord with respect to $\gamma_{1}$. Substitution of Eqs. (18), (19), and (34) into Eq. (33), the first derivative of $\mathcal{D}\left(\hat{\rho}\left(\gamma_{1}\right)\right)$ with respect to $\gamma_{1}$ has the following forms

$$
\frac{\partial \mathcal{D}\left(\hat{\rho}\left(\gamma_{1}\right)\right)}{\partial \gamma_{1}}=\left\{\begin{array}{cc}
F\left(\gamma_{1}\right)+G\left(\gamma_{1}\right), & 0<t<t_{c}, \\
G\left(\gamma_{1}\right), & t \geq t_{c}
\end{array}\right.
$$

where we have introduced the following three functions

$$
\begin{aligned}
& F\left(\gamma_{1}\right)=\frac{\left|c_{1}-c_{2}\right|+|h|}{4} \log _{2}\left(\frac{2-|\mu|-|v|}{2+|\mu|+|v|}\right), \\
& G\left(\gamma_{1}\right)=\frac{c_{1}-c_{2}}{4} \log _{2}\left(\frac{1+c_{3}+\mu}{1+c_{3}-\mu}\right)+\frac{h}{4} \log _{2}\left(\frac{1-c_{3}+v}{1-c_{3}-v}\right), \\
& h\left(\gamma_{1}\right)=\frac{v}{\gamma_{1}}\left(\frac{r-1}{r+1}\right)^{2} .
\end{aligned}
$$

For the initial state of $0 \leq c_{2}<c_{1}<1$ and $\left|c_{3}\right|<1$, from Eq. (49) we can see that the function $G\left(\gamma_{1}\right)$ is always positive. This implies that when $t>t_{c}$ the derivative of the quantum discord with respect to the time is always negative, i.e., $\partial \mathcal{D}\left(\hat{\rho}\left(\gamma_{1}\right)\right) / \partial t<0$. Hence, the quantum discord always decreases monotonically when $t>t_{c}$. That is, it is impossible to amplify the prepared quantum discord in the time evolution after the critic time $t_{c}$.

On the other hand, from Eqs. (48) and (49) we can see that the function $F\left(\gamma_{1}\right)$ is always negative due to $(2-|\mu|-|v|) /(2+$ $|\mu|+|v|)<1$. Then, $\partial \mathcal{D}\left(\hat{\rho}\left(\gamma_{1}\right)\right) / \partial \gamma_{1}<0$, i.e., $\partial \mathcal{D}\left(\hat{\rho}\left(\gamma_{1}\right)\right) / \partial t<$ 0 , is possible due to the competing change of the two functions $F\left(\gamma_{1}\right)$ and $G\left(\gamma_{1}\right)$ in the time evolution when $t<t_{c}$. Therefore, it is possible to amplify the initially prepared quantum discord before the critic time.

We now investigate numerically the discord amplification for certain initially prepared $X$-type states of two different qubits. In Fig. 6 we display the dynamic evolution of the quantum discord at zero temperature. Here the two qubits are initially prepared in the $X$-type state with $c_{1}=0.6, c_{2}=0$, and $c_{3}=0.3$, the detuning parameter $r=1,1.5,2$, and 5 , respectively. The solid black line corresponds to the case of two identical qubits discussed in the previous subsection. In this case, the initially prepared discord can be amplified and this discord amplification is stable with the critic time $t_{c}=\infty$. From Fig. 6 we can see that the detuning of the two qubits described by the parameter $r$ can affect seriously the discord evolution. The critic time at which the sudden change of the discord happens becomes shorten with the increase of the detuning parameter $r$. After the critic time, the discord begins to decay asymptotically. There exists an amplification regime of the discord before the critic time. The amplification regime becomes narrow with the increase of $r$, and the amplification disappears when the detuning is large enough.

The above discussions on the discord dynamics inspire us to ask an interesting question: may the discord remain unchange in the time evolution before the critic time for certain cases? In the following we show that it is possible to maintain the discord before the critic time. In order to see this, we consider two different qubits with a large detuning $r \gg 1$, which are initially prepared $X$-type states with the three state parameters $c_{1}=1$, and $0<-c_{2}=c_{3}=c<1$. In this case, at zero temperature we find the critic time is given by

$$
t_{c}=\frac{1}{\omega_{c}} \sqrt{c^{-2 /\left(\eta \omega_{A}^{2}\right)}-1},
$$

and the quantum discord has the following expressions

$$
\mathcal{D}(\hat{\rho})=\left\{\begin{array}{c}
\frac{1+c}{2} \log _{2}(1+c)+\frac{1-c}{2} \log _{2}(1-c), \quad t<t_{c}, \\
\frac{1+\gamma_{1}}{2} \log _{2}\left(1+\gamma_{1}\right)+\frac{1-\gamma_{1}}{2} \log _{2}\left(1-\gamma_{1}\right), t \geq t_{c},
\end{array}\right.
$$

where $\gamma_{1}(t)$ is given by Eq. (43). From Eq. (51) we can see that before the critic time the quantum discord is independent of the time. This indicates that the initial discord of the $X$-type states under present consideration does not change in the time evolution before the critic time $t_{c}$. Hence, the environment does not affect the quantum discord in this time evolution regime. In other words, the quantum discord is decoherence-free for such $X$-type states with the three state parameters $c_{1}=1$, and $0<-c_{2}=c_{3}=c<1$ in the time evolution before the critic time. From Eq. (50) we can see that the smaller the value of the state parameter $c$ the longer the discord can remain unchanged.

\section{CONCLUDING REMARKS}

In conclusion, we have studied the quantum discord dynamics of two uncoupled qubits immersed in a common heat bath. This system is depicted by an exactly solvable phase decoherence model. We have shown that the quantum discord of two noninteracting qubits can be amplified or protected for certain initially prepared $X$-type states in the time evolution. Especially, it has been found that there does exist the stable amplification of the quantum discord for certain $X$-type states 
when the two qubits are identical, and the quantum discord can be protected for the case of two different qubits with a large detuning. However, the stable amplification cannot be created when the two qubits are not identical for the same $X$ type states. The degree of the discord amplification decreases with the increase of the detuning between the two qubits. The amplification and protection of the quantum discord can be understood as quantum effects induced by the environment since the two qubits interact neither directly, nor with a third system, but with the common reservoir with infinitely many degrees of freedoms. Both of them are decoherence free. The stable amplification (protection) of the quantum discord can also be considered as a resonant (large-detuning) effect of the two qubits interacting with the common reservoir.

It has been indicated that in general there does exist a sudden change of the quantum discord for the two qubits in the time evolution at a critic time point $t_{c}$, and the discord amplification and protection may occur only in the time interval $0<t \leq t_{c}$ for certain $X$-type states. Generally, the critic time depends on the initial-state parameters, characteristic parameters of the two qubits and their environment, and the qubitreservoir interaction. The quantum discord will decay asymptotically to zero after the critic time. The creation of the stable amplification and protection of the quantum discord for the two qubits in a heat bath sheds new light on production of quantum states with long-living quantum discord.

\section{Acknowledgments}

This work was supported by NFRP of China Grant No. 2007CB925204, NSF of China Grant No. 10775048, PCSIRT Grant No. IRT0964, and ECHP Grant No. 08W012. J. B. Yuan thanks Qiong Wang, Qin-Shou Tan, Mi Jiang, and Yu-Xia Shu for useful discussions.
[1] B. Schumacher and M. D. Westmoreland, Phys. Rev. A 74, 042305 (2006).

[2] B. Groisman, S. Popescu, and A. Winter, Phys. Rev. A 72, 032317 (2005).

[3] L. Henderson and V. Vedral, J. Phys. A 34, 6899 (2001); V. Vedral, Phys. Rev. Lett. 90, 050401 (2003).

[4] J. Oppenheim, M. Horodecki, P. Horodecki, and R. Horodecki, Phys. Rev. Lett. 89, 180402 (2002).

[5] D. Yang, M. Horodecki, and Z. D. Wang, Phys. Rev. Lett. 101, 140501 (2008).

[6] D. L. Zhou, Phys. Rev. Lett. 101, 180505 (2008).

[7] H. Ollivier and W. H. Zurek, Phys. Rev. Lett. 88, 017901 (2001).

[8] D. Kaszlikowski, A. Sen(De), U. Sen, V. Vedral, and A. Winter, Phys. Rev. Lett. 101, 070502 (2008).

[9] M. Piani, P. Horodecki, and R. Horodecki, Phys. Rev. Lett. 100, 090502 (2008); M. Piani, M. Christandl, C. E. Mora, and P. Horodecki, ibid. 102, 250503 (2009).

[10] A. Datta, A. Shaji, and C. M. Caves, Phys. Rev. Lett. 100, 050502 (2008).

[11] B. P. Lanyon, M. Barbieri, M. P. Almeida, and A. G. White, Phys. Rev. Lett. 101, 200501 (2008).

[12] E. Knill and R. Laflamme, Phys. Rev. Lett. 81, 5672 (1998).

[13] R. Dillenschneider, Phys. Rev. B 78, 224413 (2008).

[14] M. S. Sarandy, Phys. Rev. A 80, 022108 (2009).

[15] J. Cui and H. Fan, J. Phys. A: Math. Theor. 43, 045305 (2010).

[16] C. A. Rodriguez-Rosario, K. Modi, A. Kuah, A. Shaji, and E. C. G. Sudarshan, J. Phys. A: Math. Theor. 41, 205301 (2008); A. Shabani and D. A. Lidar, Phys. Rev. Lett. 102, 100402 (2009).

[17] T. Werlang and G. Rigolin, Phys. Rev. A 81, 044101 (2010).

[18] Q. Wang, J. Q. Liao, and H. S. Zeng, Chin. Phys. B, in press (2010).

[19] Y. X. Chen and Z. Yin, arXiv:1002.0176

[20] M. Ali, A. R. P. Rau, and G. Alber, Phys. Rev. A 81, 042105 (2010).

[21] S. Luo, Phys. Rev. A 77, 042303 (2008).

[22] X. M. Lu, Z. J. Xi, Z. Sun, and X. G. Wang, arXiv:1004.5281
[23] T. Werlang, S. Souza, F. F. Fanchini, and C. J. Villas Boas, Phys. Rev. A 80, 024103 (2009).

[24] J. Maziero, L. C. Céleri, R. M. Serra, and V. Vedral, Phys. Rev. A 80, 044102 (2009).

[25] B. Wang, Z. Y. Xu, Z. Q. Chen, and M. Feng, Phys. Rev. A 81, 014101 (2010); F. F. Fanchini, T. Werlang, C. A. Brasil, L. G. E. Arruda, and A. O. Caldeira, Phys. Rev. A 81, 052107 (2010).

[26] J. Maziero, T. Werlang, F. F. Fanchini, L. C. Céleri, and R. M. Serra, Phys. Rev. A 81, 022116 (2010).

[27] L. Mazzola, J. Piilo, and S. Maniscalco, Phys. Rev. Lett., in press (2010).

[28] T. Yu and J. H. Eberly, Phys. Rev. Lett. 93, 140404 (2004); Science 323, 598 (2009).

[29] B. Bellomo, R. Lo Franco, and G. Compagno, Phys. Rev. Lett. 99, 160502 (2007).

[30] J. Laurat, K. S. Choi, H. Deng, C. W. Chou, and H. J. Kimble, Phys. Rev. Lett. 99, 180504 (2007).

[31] C. E. López, G. Romero, F. Lastra, E. Solano, and J. C. Retamal, Phys. Rev. Lett. 101, 080503 (2008).

[32] A. Al-Qasimi and D. F. V. James, Phys. Rev. A 77, 012117 (2008).

[33] S. Maniscalco, F. Francica, R. L. Zaffino, N. L. Gullo, and F. Plastina, Phys. Rev. Lett. 100, 090503 (2008).

[34] A. Ferraro, L. Aolita, D. Cavalcanti, F. M. Cucchietti, and A. Acln, arXiv:0908.3157v3.

[35] T. M. Cover and J. A. Thomas, Elements of Information Theory (Wiley Interscience, New York, 2006).

[36] A. O. Caldeira and A. J. Leggett, Ann. Phys. (N.Y.) 149, 374 (1983).

[37] L. M. Kuang, H. S. Zeng, and Z. Y. Tong, Phys. Rev. A 60, 3815 (1999); L. M. Kuang, Z. Y. Tong, Z. W. Ouyang, and H. S. Zeng, Phys. Rev. A 61, 013608 (1999).

[38] S. Chakravarty and A. J. Leggett, Phys. Rev. Lett. 52, 5 (1984); A. J. Leggett, S. Chakravarty, A. T. Dorsey, M. P. A. Fisher, A. Garg, and W. Zwerger, Rev. Mod. Phys. 59, 1 (1987). 